\title{
Guided Electromagnetic Waves Propagating in a Two-Layer Cylindrical Dielectric Waveguide with Inhomogeneous Nonlinear Permittivity
}

\author{
E. Yu. Smol'kin and D. V. Valovik \\ Department of Mathematics and Supercomputing, Penza State University, Krasnaya Street 40, Penza 440026, Russia \\ Correspondence should be addressed to D. V. Valovik; dvalovik@mail.ru
}

Received 12 November 2014; Accepted 22 January 2015

Academic Editor: Ivan Avramidi

Copyright (c) 2015 E. Yu. Smol'kin and D. V. Valovik. This is an open access article distributed under the Creative Commons Attribution License, which permits unrestricted use, distribution, and reproduction in any medium, provided the original work is properly cited.

\begin{abstract}
The paper focuses on the problem of monochromatic electromagnetic TM wave propagation in a two-layer circular cylindrical dielectric waveguide. The space outside the waveguide is filled with isotropic medium having constant permittivity. The inner core of the waveguide is filled with isotropic medium having constant permittivity; the cladding of the core is filled with isotropic inhomogeneous nonlinear permittivity (the nonlinear term is expressed by Kerr law). Existence of guided modes which depend harmonically on $z$ (the waveguide axis coincides with $z$-axis) is proved and their localization is found. Numerical results including different type of nonlinearities are presented. A comparison with the linear case is given. The existence of a new propagation regime is predicted.
\end{abstract}

\section{Introduction}

The paper studies the problem of monochromatic electromagnetic TM (transverse-magnetic) wave propagation in a two-layered circle cylindrical dielectric waveguide with nonlinear permittivity inside one of its layers. Here we talk only about intensity-dependent permittivity. We do not consider multiple harmonic generation or other nonlinear effects that in rigorous statement involve time-dependent Maxwell's equations. The nonlinear permittivity is described by the Kerr law. Kerr law is one of the most important dependencies in nonlinear optics; see, for example, [1-3], and for newest experimental observation see [4-6].

The physical problem is reduced to a nonlinear transmission eigenvalue problem for a system of nonlinear ordinary differential equations. Eigenvalues of the problem correspond to propagation constants (PCs) of the waveguide. The full set of PCs of a waveguide is one of the most important characteristics of the waveguide; this characteristic is used for waveguide's designing. One of the main methods to study the problem is the small parameter method. Since the Kerr law is characterised by a small constant factor in front of the nonlinear term (coefficient of the nonlinearity), then this approach is justified. Numerical results are based on a numerical method that does not depend on the smallness of the parameter [7]. As is known the Kerr law is described by an unbounded function; in order to demonstrate difference between unbounded and bounded nonlinear permittivities we presented numerical results for both cases; we also gave a comparison between linear and nonlinear cases. Numerical results given here demonstrate not only those eigenvalues that are predicted by the main theorem of this work but new eigenvalues that correspond to a new nonlinear propagation regime.

Currently there has been significant progress in studying of polarised electromagnetic TE and TM wave propagation in waveguide structures (for a planar waveguide, see [8-11]; for a circular cylindrical waveguide, see $[1,8,12-15]$; for a circular two-layered cylindrical waveguide, see $[7,16])$ filled with nonlinear dielectric permittivities. In particular, theorems on existence and localization of eigenvalues in some of these problems have been proved.

Most of these papers are devoted to studying of polarized waves in waveguides filled with a homogeneous nonlinear 


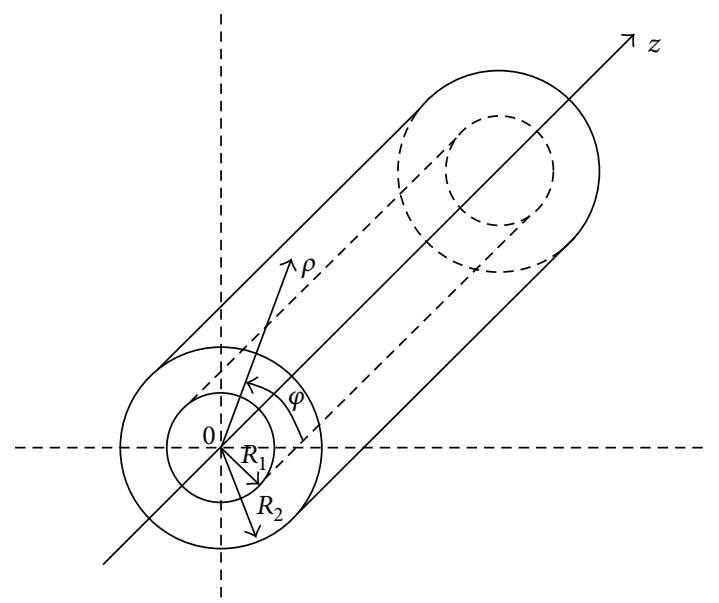

FIgURE 1: Geometry of the problem.

medium. From aforementioned studies only [7, 13, 15, 16] focus on inhomogeneous nonlinear permittivity.

Multilayered cylindrical linear homogeneous waveguide was studied in $[17,18]$, and one of the practical applications for nonlinear two-layered waveguides is shown in [19].

Results obtained in this paper together with the results given in [16] give an opportunity to consider a very intriguing phenomenon of coupled electromagnetic TE-TM wave propagation in a two-layered cylindrical waveguide. This problem will be treated in a separate paper. For different types of phenomena of coupled wave propagation see [20-22].

\section{Governing Equations}

Consider three-dimensional space $\mathbb{R}^{3}$ with cylindrical coordinate system $O \rho \varphi z$. In this space a two-layer circular cylindrical waveguide

$$
\begin{aligned}
\Sigma & :=\left\{(\rho, \varphi, z): 0 \leqslant \rho<R_{1}, 0 \leqslant \varphi<2 \pi\right\} \\
& \cup\left\{(\rho, \varphi, z): R_{1} \leqslant \rho \leqslant R_{2}, 0 \leqslant \varphi<2 \pi\right\}
\end{aligned}
$$

is placed; the waveguide axis coincides with $\mathrm{Oz}$. (The waveguide is unlimited in $z$ direction.) The waveguide is filled with isotropic inhomogeneous nonlinear medium. The space outside $\Sigma$ is filled with isotropic medium characterised by constant permittivity. Throughout the paper we assume that everywhere $\mu=\mu_{0}$, where $\mu_{0}>0$ is the permeability of free space. Geometry of the problem is shown in Figure 1.

Consider monochromatic electromagnetic field

$$
\widetilde{\mathbf{E}}=\operatorname{Re}\left\{\mathbf{E} e^{-i \omega t}\right\}, \quad \widetilde{\mathbf{H}}=\operatorname{Re}\left\{\mathbf{H} e^{-i \omega t}\right\}
$$

propagating at a frequency $\omega$ along the surface of $\Sigma$. The quantities $\mathbf{E}, \mathbf{H}$ are called the complex amplitudes [1]; $\mathbf{E}=$ $\left(E_{\rho}, E_{\varphi}, E_{z}\right)^{\mathrm{T}}$ and $\mathbf{H}=\left(H_{\rho}, H_{\varphi}, H_{z}\right)^{\mathrm{T}}$, where $(\cdot)^{\mathrm{T}}$ denotes the transpose operation.
Complex amplitudes E, $\mathbf{H}$ of a monochromatic electromagnetic field must satisfy time-harmonic Maxwell's equations

$$
\begin{aligned}
& \operatorname{rot} \mathbf{H}=-i \omega \varepsilon \mathbf{E}, \\
& \operatorname{rot} \mathbf{E}=i \omega \mu \mathbf{H},
\end{aligned}
$$

the continuity conditions for the tangential components of the field at the interfaces (on the boundary of the waveguide) $\rho=R_{1}, \rho=R_{2}$, and the radiation condition at infinity: the electromagnetic field decays as $O\left(|\rho|^{-1}\right)$ when $\rho \rightarrow \infty$. The solutions to Maxwell's equations are sought in the entire space.

In the whole space the dielectric permittivity has the form $\varepsilon=\widetilde{\varepsilon} \varepsilon_{0}$, where

$$
\widetilde{\varepsilon}= \begin{cases}\varepsilon_{1}, & 0 \leqslant \rho<R_{1}, \\ \varepsilon_{2}(\rho)+\alpha|\mathbf{E}|^{2}, & R_{1} \leqslant \rho \leqslant R_{2}, \\ \varepsilon_{3}, & \rho>R_{2},\end{cases}
$$

$\varepsilon_{1}, \varepsilon_{3}$ are positive real constants, $\varepsilon_{0}>0$ is the permittivity of free space, and

$$
|\mathbf{E}|^{2}=\left|\left(\mathbf{E} e^{-i \omega t}, \mathbf{e}_{\rho}\right)\right|^{2}+\left|\left(\mathbf{E} e^{-i \omega t}, \mathbf{e}_{\varphi}\right)\right|^{2}+\left|\left(\mathbf{E} e^{-i \omega t}, \mathbf{e}_{z}\right)\right|^{2}
$$

here $\mathbf{e}_{k}$ is an orthonormal vector in $k$ direction, $(\cdot, \cdot)$ is the Euclidean scalar product, $\varepsilon_{2}(\rho) \in C^{1}\left[R_{1}, R_{2}\right], \varepsilon_{2}(\rho)>0$, and $\alpha>0$ is a real constant.

Note 1. Inside $\Sigma$ the quantities $\varepsilon_{1}$ and $\varepsilon_{2}+\alpha|\mathbf{E}|^{2}$ are scalars. It is possible to consider an anisotropic case in which one of these quantities (or both) is a diagonal tensor.

\section{Statement of the Problem}

Consider TM-polarized waves in the monochromatic mode

$$
\begin{aligned}
& \mathbf{E} e^{-i \omega t}=e^{-i \omega t}\left(E_{\rho}, 0, E_{z}\right)^{\mathrm{T}}, \\
& \mathbf{H} e^{-i \omega t}=e^{-i \omega t}\left(0, H_{\varphi}, 0\right)^{\mathrm{T}} .
\end{aligned}
$$

It is assumed that waves propagating along the boundary of $\Sigma$ depend harmonically on $z[1,8,16,23]$. Substituting the TM waves into (3) one is convinced that the field does not depend on $\varphi$. Thus the components of the field have the form

$$
\begin{gathered}
E_{\rho}=\mathrm{E}_{\rho}(\rho) e^{i \gamma z}, \quad E_{z}=\mathrm{E}_{z}(\rho) e^{i \gamma z} \\
H_{\varphi}=\mathrm{H}_{\varphi}(\rho) e^{i \gamma z}
\end{gathered}
$$

where $\gamma$ is a real spectral parameter of the problem which defines unknown PCs (without loss of generality we suppose $\gamma>0$ ). In what follows arguments of functions will often be omitted. 
Let $k_{0}^{2}:=\omega^{2} \mu_{0} \varepsilon_{0}$. Substituting the complex amplitudes E, $\mathbf{H}$ with components (7) into Maxwell's equations (3) one obtains

$$
\begin{gathered}
i \gamma \mathrm{E}_{\rho}-\mathrm{E}_{z}^{\prime}=i \omega \mu \mathrm{H}_{\varphi}, \\
i \gamma \mathrm{H}_{\varphi}=i \omega \varepsilon \mathrm{E}_{\rho}, \\
\rho^{-1}\left(\rho \mathrm{H}_{\varphi}\right)^{\prime}=-i \omega \varepsilon \mathrm{E}_{z} .
\end{gathered}
$$

Introducing the notations $u_{1}(\rho ; \gamma):=\mathrm{E}_{\rho}(\rho ; \gamma)$ and $u_{2}(\rho ; \gamma):=$ $i \mathrm{E}_{z}(\rho ; \gamma)$ one obtains from (8) that

$$
\begin{gathered}
\left(\gamma^{2}-k_{0}^{2} \widetilde{\varepsilon}\right) u_{1}+\gamma u_{2}^{\prime}=0, \\
\gamma\left(\rho u_{1}\right)^{\prime}+\left(\rho u_{2}^{\prime}\right)^{\prime}+k_{0}^{2} \widetilde{\varepsilon} \rho u_{2}=0,
\end{gathered}
$$

where $u_{1}, u_{2}$ are real functions and $\gamma^{2}>k_{0}^{2} \max \left\{\varepsilon_{1}, \varepsilon_{3}\right\}$.

It is also assumed that functions $u_{1}, u_{2}$ are sufficiently smooth:

$$
\begin{aligned}
u_{1} & \in C\left[0, R_{1}\right] \cap C\left[R_{1}, R_{2}\right] \cap C\left[R_{2},+\infty\right) \\
& \cap C^{1}\left(0, R_{1}\right) \cap C^{1}\left(R_{1}, R_{2}\right) \cap C^{1}\left(R_{2},+\infty\right), \\
u_{2} & \in C[0,+\infty) \cap C^{1}\left[0, R_{1}\right] \cap C^{1}\left[R_{1}, R_{2}\right] \cap C^{1}\left[R_{2},+\infty\right) \\
& \cap C^{2}\left(0, R_{1}\right) \cap C^{2}\left(R_{1}, R_{2}\right) \cap C^{2}\left(R_{2},+\infty\right) .
\end{aligned}
$$

Tangential components of electromagnetic field are known to be continuous at interfaces $[23,24]$. Taking into account that the tangential components are $E_{z}$ and $H_{\varphi}$ and using formulae (8) one obtains

$$
\begin{array}{ll}
{\left.\left[\widetilde{\mathcal{\varepsilon}} u_{1}\right]\right|_{\rho=R_{1}}=0,} & {\left.\left[u_{2}\right]\right|_{\rho=R_{1}}=0,} \\
{\left.\left[\widetilde{\mathcal{\varepsilon}} u_{1}\right]\right|_{\rho=R_{2}}=0,} & {\left.\left[u_{2}\right]\right|_{\rho=R_{2}}=0,}
\end{array}
$$

where $\left.[v]\right|_{\rho=s}=\lim _{\rho \rightarrow s-0} v(\rho)-\lim _{\rho \rightarrow s+0} v(\rho)$.

Problem $P_{\mathrm{M}}(\alpha)$ is to prove existence of quantities $\hat{\gamma}$ such that for prescribed value of the field at a boundary of $\Sigma$ there exist nontrivial functions $u_{1}(\rho ; \hat{\gamma}), u_{2}(\rho ; \hat{\gamma})$ defined for $\rho \in$ $[0,+\infty)$, which must satisfy system (9), conditions (10), (11), and the radiation condition at infinity.

The quantities $\widehat{\gamma}$ solving problem $P_{\mathrm{M}}(\alpha)$ are called eigenvalues or PCs; corresponding functions $u_{1}(\rho ; \widehat{\gamma}), u_{2}(\rho ; \widehat{\gamma})$ are called eigenfunctions (see, e.g., $[25,26])$.

\section{Main Nonlinear Equations}

Taking into account the boundedness condition of the field in any finite region and the radiation condition at infinity solutions to (9) outside the cladding have the form

$$
\begin{gathered}
u_{1}(\rho)= \begin{cases}-C_{1} \gamma k_{1}^{-1} I_{1}\left(k_{1} \rho\right), & 0 \leqslant \rho<R_{1}, \\
C_{2} \gamma k_{3}^{-1} K_{1}\left(k_{3} \rho\right), & \rho>R_{2} ;\end{cases} \\
u_{2}(\rho)= \begin{cases}C_{1} I_{0}\left(k_{1} \rho\right), & 0 \leqslant \rho<R_{1}, \\
C_{2} K_{0}\left(k_{3} \rho\right), & \rho>R_{2},\end{cases}
\end{gathered}
$$

where $k_{1}^{2}:=\gamma^{2}-k_{0}^{2} \varepsilon_{1}, k_{3}^{2}:=\gamma^{2}-k_{0}^{2} \varepsilon_{3} ; I_{0}, I_{1}, K_{0}$, and $K_{1}$ are the modified Bessel functions [27]. The constant $C_{1}$ is assumed to be known (see the definition of $P_{\mathrm{M}}(\alpha)$ ); the constant $C_{2}$ is determined using conditions (11). Solutions (12) are real as $\gamma^{2}>k_{0}^{2} \max \left\{\varepsilon_{1}, \varepsilon_{3}\right\}$.

Let

$$
\begin{gathered}
L_{M}:=\frac{d}{d \rho}\left(p \frac{d}{d \rho}\right)+\varepsilon_{2} \rho, \\
W:=\gamma\left(q|\mathbf{u}|^{2} u_{1}\right)^{\prime}-\rho|\mathbf{u}|^{2} u_{2},
\end{gathered}
$$

where

$$
\begin{gathered}
k_{2}^{2}(\rho):=k_{0}^{2} \varepsilon_{2}(\rho)-\gamma^{2}, \quad p(\rho):=\frac{\varepsilon_{2}(\rho) \rho}{k_{2}^{2}(\rho)}, \\
q(\rho):=\frac{\rho}{k_{2}^{2}(\rho)}, \quad|\mathbf{u}|^{2}=u_{1}^{2}+u_{2}^{2} .
\end{gathered}
$$

Thus system (9) in the waveguide cladding can be written in the form

$$
\begin{gathered}
u_{1}=k_{2}^{-2}(\rho)\left(\gamma u_{2}^{\prime}-\alpha k_{0}^{2}|\mathbf{u}|^{2} u_{1}\right), \\
L_{\mathrm{M}} u_{2}=\alpha W .
\end{gathered}
$$

In the Kerr law the coefficient $\alpha$ is supposed to be small $[2,3,28]$; for this reason the small parameter method can be used to study the problem $P_{\mathrm{M}}(\alpha)$.

For proving existence of solutions to $P_{\mathrm{M}}(\alpha)$ it is necessary at first to prove existence of solutions to the linear problem $P_{\mathrm{M}}(0)$, which is $P_{\mathrm{M}}(\alpha)$ for $\alpha=0$.

\section{Existence of Solutions to $P_{\mathrm{M}}(0)$}

The problem $P_{\mathrm{M}}(0)$ derived from $P_{\mathrm{M}}(\alpha)$ is difficult to investigate. For this reason we revert to (8) and consider the original problem for $\alpha=0$. The problem obtained in such a way is also denoted as $P_{\mathrm{M}}(0)$.

Let $\lambda:=\gamma^{2}$. Introduce the notation $u_{3}:=\rho \mathrm{H}_{\varphi}$; from system (8) for $\alpha=0$ one obtains

$$
L u_{3}=0, \quad \rho \in\left[R_{1}, R_{2}\right],
$$

where $L:=(d / d \rho)\left(\left(1 / \varepsilon_{2}(\rho) \rho\right) \cdot(d / d \rho)\right)+k_{0}^{2} / \rho-\lambda / \varepsilon_{2}(\rho) \rho$.

As the components $H_{\varphi}$ and $E_{z}$ are continuous at the interfaces one obtains the following conditions for $u_{3}$ :

$$
\begin{array}{ll}
{\left.\left[u_{3}\right]\right|_{\rho=R_{1}}=0,} & {\left.\left[u_{3}\right]\right|_{\rho=R_{2}}=0,} \\
{\left.\left[u_{3}^{\prime}\right]\right|_{\rho=R_{1}}=0,} & {\left.\left[u_{3}^{\prime}\right]\right|_{\rho=R_{2}}=0,}
\end{array}
$$

and in view of (12)

$$
u_{3}(\rho)= \begin{cases}-C_{1} \omega \varepsilon_{0} \varepsilon_{1} k_{1}^{-1} \rho I_{1}\left(k_{1} \rho\right), & 0 \leqslant \rho<R_{1}, \\ C_{2} \omega \varepsilon_{0} \varepsilon_{3} k_{3}^{-1} \rho K_{1}\left(k_{3} \rho\right), & \rho>R_{2} .\end{cases}
$$

Taking into account conditions (17) and solutions (18) one obtains boundary conditions of the third kind (which depend nonlinearly on the spectral parameter):

$$
\begin{gathered}
u_{3}^{\prime}\left(R_{1}\right)-g_{1}(\lambda) u_{3}\left(R_{1}\right)=0, \\
u_{3}^{\prime}\left(R_{2}\right)+g_{2}(\lambda) u_{3}\left(R_{2}\right)=0,
\end{gathered}
$$


where the functions $g_{1}, g_{2}$ are easily found. Thus the problem $P_{\mathrm{M}}(0)$ is a (linear) problem of Sturm-Liouville type for (16) with conditions (19).

At first one needs to consider yet another Sturm-Liouville:

$$
L v=0, \quad v^{\prime}\left(R_{1}\right)=v^{\prime}\left(R_{2}\right)=0 .
$$

Let $\left\{\lambda_{n}, v_{n}(\rho)\right\}_{n=1}^{\infty}$ be the complete system of eigenvalues and eigenfunctions orthonormal in the space $L_{2}\left(R_{1}, R_{2}\right.$; $r(\rho)$ ) with weight $r(\rho):=1 / \varepsilon_{2} \rho$ of boundary value problem (20). It is known [29] that all the eigenvalues are real and simple (of multiplicity 1). To be more precise, there are not more than a finite number of positive eigenvalues and an infinite number of negative ones $\left(\lambda_{n} \rightarrow-\infty\right.$ for $\left.n \rightarrow \infty\right)$. We arrange eigenvalues in the ascending order: $\cdots<\lambda_{i+1}<$ $\lambda_{i}<\cdots<\lambda_{2}<\lambda_{1}$.

Solvability of $P_{\mathrm{M}}(0)$ is established by the following statement (see proof in [29]). This statement asserts that required eigenvalues of $P_{M}(0)$ lie between eigenvalues of problem (20).

Statement 1. Let $\varepsilon_{2}(\rho) \in C^{1}\left[R_{1}, R_{2}\right], \varepsilon_{2}(\rho)>0$ for $\rho \in$ $\left[R_{1}, R_{2}\right]$, and problem (20) have $m+1$ positive eigenvalues $\lambda_{j}$, where $m \geqslant 1(j=\overline{1, m+1})$, such that

$$
0<k_{0}^{2} \max \left\{\varepsilon_{1}, \varepsilon_{3}\right\}<\lambda_{m+1}<\lambda_{m}<\cdots<\lambda_{2}<\lambda_{1} .
$$

Then there exist at least $m$ simple eigenvalues $\lambda_{i}^{*}>0(i=$ $\overline{1, m})$ of the problem $P_{\mathrm{M}}(0)$.

In this case PCs $\widetilde{\gamma}_{i}$ of guided TM waves in $\Sigma$ are defined as $\widetilde{\gamma}_{i}=\sqrt{\lambda_{i}^{*}} \in\left(\sqrt{\lambda_{i+1}}, \sqrt{\lambda_{i}}\right), i=\overline{1, m}$.

Note 2. Thickness $R_{2}-R_{1}$ of the waveguide cladding can be chosen in such a way that the problem $P_{\mathrm{M}}(0)$ will have solutions.

\section{Nonlinear Integral Equations and Problem $P_{\mathrm{M}}(\alpha)$}

Let $\lambda:=\gamma^{2}$. Consider the boundary value problem

$$
L_{\mathrm{M}} v=0, \quad v\left(R_{1}\right)=v\left(R_{2}\right)=0, \quad \rho \in\left[R_{1}, R_{2}\right] .
$$

Since all the coefficients of $L_{\mathrm{M}} v=0$ are continuous and nonzero on the segment $\left[R_{1}, R_{2}\right]$, then the equation has two linearly independent continuous solutions defined on the same segment. Furthermore, it is clear that the eigenvalues of problem (22) do not coincide with the eigenvalues of the problem $P_{\mathrm{M}}(0)$. Thus one obtains that a unique Green's function $G_{\mathrm{M}}(\rho, s)$ of the boundary value problem

$$
\begin{array}{r}
L_{\mathrm{M}} G_{\mathrm{M}}=-\delta(\rho-s), \quad G_{\mathrm{M}}\left(R_{1}, s\right)=G_{\mathrm{M}}\left(R_{2}, s\right)=0 \\
\left(R_{1} \leqslant \rho, s \leqslant R_{2}\right)
\end{array}
$$

exists in a neighbourhood of each eigenvalue of $P_{\mathrm{M}}(0)$.
For brevity we use the following notation:

$$
\begin{aligned}
& G_{\mathrm{M}}^{\prime}(A, s):=\left.\partial_{\rho} G_{\mathrm{M}}(\rho, s)\right|_{\rho=A}, \\
& G_{\mathrm{M}}^{\prime \prime}(A, B):=\left.\partial_{s \rho}^{2} G_{\mathrm{M}}(\rho, s)\right|_{\substack{\rho=A \\
s=B}}, \\
& G_{\mathrm{M}}^{\prime}(\rho, A):=\left.\partial_{s} G_{\mathrm{M}}(\rho, s)\right|_{s=A}, \\
& G_{\mathrm{M}}^{\prime \prime}(A, s):=\left.\partial_{s \rho}^{2} G_{\mathrm{M}}(\rho, s)\right|_{\rho=A}, \\
& G_{\mathrm{M}}^{\prime \prime}(\rho, A):=\left.\partial_{s \rho}^{2} G_{\mathrm{M}}(\rho, s)\right|_{s=A} ;
\end{aligned}
$$

for the value of $f$ at the point $A \pm 0$ we often write $f(A)$ because it is clear from the context whether it is the left or right limit; the symbol $G_{M}$ always means $G_{M}(\rho, s)$ (if necessary the dependence on $\gamma$ is explicitly indicated).

Using the second Green's formula one obtains

$$
\int_{R_{1}}^{R_{2}}\left(v L_{\mathrm{M}} u-u L_{\mathrm{M}} v\right) d \rho=\left.p(\rho)\left(u^{\prime} v-u v^{\prime}\right)\right|_{R_{1}} ^{R_{2}} .
$$

Let $v=G_{\mathrm{M}}$. Using (23), (25) one finds

$$
\begin{aligned}
& \int_{R_{1}}^{R_{2}}\left(G_{\mathrm{M}} L_{\mathrm{M}} u-u L_{\mathrm{M}} G_{\mathrm{M}}\right) d \rho \\
& \quad=p\left(R_{1}\right) u\left(R_{1}\right) G_{\mathrm{M}}^{\prime}\left(R_{1}, s\right)-p\left(R_{2}\right) u\left(R_{2}\right) G_{\mathrm{M}}^{\prime}\left(R_{2}, s\right) .
\end{aligned}
$$

From the previous formula one obtains the integral representation of a solution $u_{2}(s)$ to system (15):

$$
\begin{aligned}
u_{2}(s)= & -\alpha \int_{R_{1}}^{R_{2}} G_{\mathrm{M}} W d \rho+p\left(R_{1}\right) u_{2}\left(R_{1}\right) G_{\mathrm{M}}^{\prime}\left(R_{1}, s\right) \\
& -p\left(R_{2}\right) u_{2}\left(R_{2}\right) G_{\mathrm{M}}^{\prime}\left(R_{2}, s\right) .
\end{aligned}
$$

Using solutions (12), the second column of transmission conditions (11), and integrating by parts one gets from the previous formula

$$
\begin{aligned}
u_{2}(s)= & \alpha \gamma \int_{R_{1}}^{R_{2}} \partial_{\rho} G_{\mathrm{M}} \cdot q|\mathbf{u}|^{2} u_{1} d \rho \\
& +\alpha \int_{R_{1}}^{R_{2}} G_{\mathrm{M}} \cdot \rho|\mathbf{u}|^{2} u_{2} d \rho+h_{2}(s),
\end{aligned}
$$

where

$$
\begin{aligned}
h_{2}(s):= & C_{1} p\left(R_{1}\right) I_{0}\left(k_{1} R_{1}\right) G_{\mathrm{M}}^{\prime}\left(R_{1}, s\right) \\
& -C_{2} p\left(R_{2}\right) K_{0}\left(k_{3} R_{2}\right) G_{\mathrm{M}}^{\prime}\left(R_{2}, s\right) .
\end{aligned}
$$


The following calculation is required below:

$$
\begin{aligned}
\frac{\partial}{\partial s} \int_{R_{1}}^{R_{2}} \partial_{\rho} G_{\mathrm{M}} \cdot q|\mathbf{u}|^{2} u_{1} d \rho & \frac{\partial}{\partial s}\left(\int_{R_{1}}^{s-0} \partial_{\rho} G_{\mathrm{M}} \cdot q|\mathbf{u}|^{2} u_{1} d \rho+\int_{s+0}^{R_{2}} \partial_{\rho} G_{\mathrm{M}} \cdot q|\mathbf{u}|^{2} u_{1} d \rho\right) \\
= & \int_{R_{1}}^{R_{2}} \partial_{s \rho}^{2} G_{\mathrm{M}} \cdot q|\mathbf{u}|^{2} u_{1} d \rho+q(s)|\mathbf{u}(s)|^{2} u_{1}(s) \\
& \cdot\left(\left.\partial_{\rho} G_{\mathrm{M}}\right|_{\rho=s-0}-\left.\partial_{\rho} G_{\mathrm{M}}\right|_{\rho=s+0}\right) \\
= & \int_{R_{1}}^{R_{2}} \partial_{s \rho}^{2} G_{\mathrm{M}} \cdot q|\mathbf{u}|^{2} u_{1} d \rho-\frac{|\mathbf{u}(s)|^{2} u_{1}(s)}{\varepsilon_{2}(s)} .
\end{aligned}
$$

Using the previous formula one obtains from (28)

$$
\begin{aligned}
u_{2}^{\prime}(s)= & \alpha \gamma \int_{R_{1}}^{R_{2}} \partial_{s \rho}^{2} G_{\mathrm{M}} \cdot q|\mathbf{u}|^{2} u_{1} d \rho \\
& +\alpha \int_{R_{1}}^{R_{2}} \partial_{s} G_{\mathrm{M}} \cdot \rho|\mathbf{u}|^{2} u_{2} d \rho \\
& -\alpha \gamma \frac{|\mathbf{u}(s)|^{2} u_{1}(s)}{\varepsilon_{2}(s)}+h_{2}^{\prime}(s) .
\end{aligned}
$$

Using this expression one finds an integral representation for a solution $u_{1}$ to system (15). Combining these results one finally obtains

$$
\begin{aligned}
u_{1}(s)= & \frac{\alpha \gamma^{2}}{k_{2}^{2}(s)} \int_{R_{1}}^{R_{2}} \partial_{s \rho}^{2} G_{\mathrm{M}} \cdot q|\mathbf{u}|^{2} u_{1} d \rho \\
& +\frac{\alpha \gamma}{k_{2}^{2}(s)} \int_{R_{1}}^{R_{2}} \partial_{s} G_{\mathrm{M}} \cdot \rho|\mathbf{u}|^{2} u_{2} d \rho \\
& -\frac{\alpha}{k_{2}^{2}(s)}\left(\frac{\gamma^{2}}{\varepsilon_{2}(s)}+k_{0}^{2}\right)|\mathbf{u}(s)|^{2} u_{1}(s)+h_{1}(s), \\
u_{2}(s)= & \alpha \gamma \int_{R_{1}}^{R_{2}} \partial_{\rho} G_{\mathrm{M}} \cdot q|\mathbf{u}|^{2} u_{1} d \rho \\
& +\alpha \int_{R_{1}}^{R_{2}} G_{\mathrm{M}} \cdot \rho|\mathbf{u}|^{2} u_{2} d \rho+h_{2}(s),
\end{aligned}
$$

where $R_{1} \leqslant s \leqslant R_{2} ; h_{1}(s):=\left(\gamma / k_{2}^{2}(s)\right) h_{2}^{\prime}(s)$.

Rewrite system (32) in an operator form. Introduce a linear operator

$$
\mathbf{N}:=\mathbf{K}+\mathbf{J},
$$

where the linear operator $\mathbf{J}$ is defined by the formula

$$
\mathbf{J}=-\frac{1}{k_{2}^{2}(s)}\left(\frac{\gamma^{2}}{\varepsilon_{2}(s)}+k_{0}^{2}\right)\left(\begin{array}{ll}
1 & 0 \\
0 & 0
\end{array}\right),
$$

the matrix linear integral operator $\mathbf{K}$ is defined by the formula

$$
\mathbf{K g}=\int_{R_{1}}^{R_{2}} \mathbf{K}(\rho, s) \mathbf{g}(\rho) d \rho,
$$

where

$$
\begin{aligned}
\mathrm{K}(\rho, s) & =\left\{K_{n m}(\rho, s)\right\}_{n, m=1}^{2} \\
& =\left(\begin{array}{cc}
\gamma^{2} q(\rho) \frac{\partial_{s \rho} G_{\mathrm{M}}}{k_{2}^{2}(s)} & \gamma \rho \frac{\partial_{s} G_{\mathrm{M}}}{k_{2}^{2}(s)} \\
\gamma q(\rho) \partial_{\rho} G_{\mathrm{M}} & \rho G_{\mathrm{M}}
\end{array}\right),
\end{aligned}
$$

and $\mathbf{g}=\left(g_{1}, g_{2}\right)^{T}$.

System (32) can be rewritten as

$$
\mathbf{u}=\alpha \mathbf{N}\left(|\mathbf{u}|^{2} \mathbf{u}\right)+\mathbf{h},
$$

where $\mathbf{h}=\left(h_{1}, h_{2}\right)^{T}$ is defined by formulae (29), (32).

Equation (37) is studied in $\mathrm{C}\left[R_{1}, R_{2}\right]=C\left[R_{1}, R_{2}\right] \times$ $C\left[R_{1}, R_{2}\right]$ with norm $\|\mathbf{u}\|_{\mathrm{C}}^{2}=\left\|u_{1}\right\|_{C}^{2}+\left\|u_{2}\right\|_{C}^{2}$, where $\|u\|_{C}=$ $\max _{x \in\left[R_{1}, R_{2}\right]}|u(x)|$.

It should be noted that (37) contains the unknown constant $C_{2}$. This constant can be expressed through $C_{1}$, which is assumed to be known (see Section 8). Expressing $C_{2}$ through $C_{1}$ and substituting it into (37) one obtains an operator $\widetilde{\mathbf{N}}$ and a vector $\widetilde{\mathbf{h}}$ instead of $\mathbf{N}$ and $\mathbf{h}$, respectively, where $\widetilde{\mathbf{N}}$ and $\widetilde{\mathbf{h}}$ are bounded. An example of how this can be done is seen, for example, in [21].

Note 3. An iterative procedure to determine approximate eigenvalues of $P_{\mathrm{M}}(\alpha)$ can be formulated and grounded. On this way one can prove convergence of approximate eigenvalues obtained at each step of the iterative procedure to exact ones. However, here we do not formulate these results, since the main purpose of the mathematics used here is to give a rigorous proof of solvability of the problem $P_{\mathrm{M}}(\alpha)$. For numerical calculations it is more convenient to use an approach based on solving an auxiliary Cauchy problem [7, $16]$.

\section{Operator Equation Studying}

It follows from general properties of Green's function that the functions $K_{i j}$ are piecewise continuous in the (closed) square $\bar{\Pi}=\left[R_{1}, R_{2}\right] \times\left[R_{1}, R_{2}\right]$. Thus the operator $\mathbf{K}$ : $\mathrm{C}\left[R_{1}, R_{2}\right] \rightarrow \mathrm{C}\left[R_{1}, R_{2}\right]$ is bounded. Obviously, the operator $\mathrm{J}: \mathrm{C}\left[R_{1}, R_{2}\right] \rightarrow \mathrm{C}\left[R_{1}, R_{2}\right]$ is also bounded.

It is easy to prove the following.

Statement 2. Let $\mathbf{K}: \mathbf{C}\left[R_{1}, R_{2}\right] \rightarrow \mathbf{C}\left[R_{1}, R_{2}\right]$ be a matrix integral operator with bounded and piecewise continuous kernels $K_{n m}(x, y)$ in the square $\bar{\Pi}$. Then the operator $\mathbf{K}$ is bounded and $\|\mathbf{K}\|_{\mathbf{C} \rightarrow \mathbf{C}} \leqslant M$, where $M^{2}=2\left(\max _{j=1,2}\left\|K_{1 j}\right\|_{\mathbf{C} \rightarrow \mathbf{C}}^{2}+\right.$ $\max _{j=1,2}\left\|K_{2 j}\right\|_{\mathbf{C} \rightarrow \mathbf{C}}^{2}$. $[8,12]$ :

Consider the algebraic cubic equation with respect to $r_{0}$

$$
\alpha\|\mathbf{N}\| r_{0}^{3}+\|\mathbf{h}\|=r_{0} .
$$

If the inequality

$$
0 \leqslant\|\mathbf{h}\|<2(3 \sqrt{3 \alpha\|\mathbf{N}\|})^{-1}
$$

holds, then (38) has two nonnegative roots $r_{*}$ and $r^{*}[8,12]$. 
The following two statements are proved in $[8,12]$.

Statement 3. If $\alpha \leqslant A^{2}$, where $A=2(3\|\mathbf{h}\| \sqrt{3\|\mathbf{N}\|})^{-2}$, then (37) has a unique continuous solution $\mathbf{u} \in \mathbf{C}\left[R_{1}, R_{2}\right]$ in the ball $B_{r_{*}} \equiv\left\{\mathbf{u}:\|\mathbf{u}\| \leqslant r_{*}\right\}$.

Note that $A$ is independent of $\alpha$ and $A>0$.

Statement 4. Let kernels of $\mathbf{N}$ and $\mathbf{h}$ in (37) depend continuously on the parameter $\gamma \in \Gamma_{0}$ on a real interval $\Gamma_{0}$ and

$$
\|\mathbf{h}(\gamma)\|<2(3 \sqrt{3 \alpha\|\mathbf{N}(\gamma)\|})^{-1}
$$

Then (37) has a unique continuous solution $\mathbf{u}(\rho ; \gamma)$ which depends continuously on $\gamma \in \Gamma_{0}$; that is, $\mathbf{u}(\rho ; \gamma) \subset \mathbf{C}\left(\left[R_{1}, R_{2}\right] \times\right.$ $\left.\Gamma_{0}\right)$.

Note 4. The operators $\mathbf{K}, \mathbf{J}$ do not coincide with operators of the same name from $[8,12]$. For this reason Statements 2-4 are not repetitions of similar statements from $[8,12]$ but they are proved in the same way.

\section{Dispersion Equation}

Using the first column of transmission conditions (11) and solutions (12) one finds

$$
\begin{aligned}
& -C_{1} \varepsilon_{1} \gamma k_{1}^{-1} I_{1}\left(k_{1} R_{1}\right) \\
& \quad=\varepsilon_{2}\left(R_{1}\right) u_{1}\left(R_{1}+0\right)+\alpha\left|\mathbf{u}\left(R_{1}+0\right)\right|^{2} u_{1}\left(R_{1}+0\right), \\
& C_{2} \varepsilon_{3} \gamma k_{3}^{-1} K_{1}\left(k_{3} R_{2}\right) \\
& \quad=\varepsilon_{2}\left(R_{2}\right) u_{1}\left(R_{2}-0\right)+\alpha\left|\mathbf{u}\left(R_{2}-0\right)\right|^{2} u_{1}\left(R_{2}-0\right) .
\end{aligned}
$$

Using the first equation from (32) the previous formulae can be rewritten in the form

$$
\begin{aligned}
& -C_{1} \varepsilon_{1} \gamma k_{1}^{-1} I_{1}\left(k_{1} R_{1}\right)=\alpha \frac{\gamma}{k_{2}^{2}\left(R_{1}\right)} F\left(R_{1}\right)+\varepsilon_{2}\left(R_{1}\right) h_{1}\left(R_{1}\right), \\
& C_{2} \varepsilon_{3} \gamma k_{3}^{-1} K_{1}\left(k_{3} R_{2}\right)=\alpha \frac{\gamma}{k_{2}^{2}\left(R_{2}\right)} F\left(R_{2}\right)+\varepsilon_{2}\left(R_{2}\right) h_{1}\left(R_{2}\right),
\end{aligned}
$$

where

$$
\begin{aligned}
F(s):= & \gamma \varepsilon_{2}(s) \int_{R_{1}}^{R_{2}} \partial_{s \rho} G_{M}(\rho, s) \cdot q|\mathbf{u}|^{2} u_{1} d \rho \\
& +\varepsilon_{2}(s) \int_{R_{1}}^{R_{2}} \partial_{s} G_{M}(\rho, s) \cdot \rho|\mathbf{u}|^{2} u_{2} d \rho \\
& -2 \gamma|\mathbf{u}(s)|^{2} u_{1}(s) .
\end{aligned}
$$

Excluding the unknown $C_{2}$ from (42) one obtains the DE in the form

$$
C_{1} g(\lambda)=\alpha Q(\lambda)
$$

where

$$
\begin{aligned}
g(\lambda):= & R_{1} R_{2} k_{1} k_{3} \varepsilon_{2}^{2}\left(R_{1}\right) \varepsilon_{2}^{2}\left(R_{2}\right) I_{0}\left(k_{1} R_{1}\right) K_{0} \\
& \cdot\left(k_{3} R_{2}\right) G_{\mathrm{M}}^{\prime \prime}\left(R_{1}, R_{2}\right) G_{\mathrm{M}}^{\prime \prime}\left(R_{2}, R_{1}\right) \\
& -\left(\varepsilon_{1} k_{2}^{4}\left(R_{1}\right) I_{1}\left(k_{1} R_{1}\right)+R_{1} k_{1} \varepsilon_{2}^{2}\left(R_{1}\right)\right. \\
& \left.\cdot I_{0}\left(k_{1} R_{1}\right) G_{\mathrm{M}}^{\prime \prime}\left(R_{1}, R_{1}\right)\right) \\
& \times\left(\varepsilon_{3} k_{2}^{4}\left(R_{2}\right) K_{1}\left(k_{3} R_{2}\right)\right. \\
Q(\lambda):= & \quad k_{1} k_{2}^{2}\left(R_{1}\right)\left(\varepsilon_{3} k_{2}^{4}\left(R_{2}\right) K_{1}\left(k_{3} R_{2}\right)+R_{2} k_{3} \varepsilon_{2}^{2}\right. \\
& \left.\cdot\left(R_{2}\right) K_{0}\left(k_{3} R_{2}\right) G_{\mathrm{M}}^{\prime \prime}\left(k_{2} R_{2}\right) G_{\mathrm{M}}^{\prime \prime}\left(R_{2}, R_{2}\right)\right), \\
& \cdot F\left(R_{1}\right)-R_{2} k_{1} k_{2}^{2}\left(R_{1}\right) k_{3} \varepsilon_{2}\left(R_{1}\right) \varepsilon_{2}\left(R_{2}\right) K_{0} \\
& \cdot\left(k_{3} R_{2}\right) G_{\mathrm{M}}^{\prime \prime}\left(R_{2}, R_{1}\right) F\left(R_{2}\right) .
\end{aligned}
$$

As is noted in Section 7 the constant $C_{2}$ can be expressed through the constant $C_{1}$ from the system (42). Taking this into account (37) can be rewritten in a form which does not contain $C_{2}$; thus all estimations indicated in the previous section are substantiated.

\section{Existence of Solutions to $P_{\mathrm{M}}(\alpha)$}

Consider the function $\Phi(\lambda) \equiv C_{1} g(\lambda)-\alpha Q(\lambda)$. If $\lambda=\hat{\lambda}$ is such that $\Phi(\hat{\lambda})=0$, then eigenvalues $\widehat{\gamma}$ of the problem $P_{\mathrm{M}}(\alpha)$ are determined from the equation $\hat{\lambda}=\widehat{\gamma}^{2}$.

For $\alpha=0$ from (44) one obtains $g(\lambda)=0$. Clearly, this equation determines eigenvalues (and only them) of the problem $P_{\mathrm{M}}(0)$. In Section 5 it is proved that $P_{\mathrm{M}}(0)$ has not more than a finite number of simple eigenvalues. Hence one obtains that the equation $g(\lambda)=0$ has not more than a finite number of simple roots (which coincide with eigenvalues of the problem $\left.P_{\mathrm{M}}(0)\right)$.

Let the problem $P_{\mathrm{M}}(0)$ have $m$ eigenvalues $\lambda_{i}^{*}$ such that

$$
k_{0}^{2} \max \left\{\varepsilon_{1}, \varepsilon_{3}\right\}<\lambda_{m}^{*}<\lambda_{m-1}^{*}<\cdots<\lambda_{2}^{*}<\lambda_{1}^{*} .
$$

Since each $\lambda_{i}^{*}$ is a simple root of the equation $g(\lambda)=0$, then for each $\lambda_{i}^{*}$ there exists a segment $\Lambda_{i}:=\left[\lambda_{i}^{*}-\delta_{i}^{\prime}, \lambda_{i}^{*}+\right.$ $\left.\delta_{i}^{\prime \prime}\right]$ such that the function $g(\lambda)$ has different signs at the endpoints of the segment (where $\delta_{i}^{\prime}>0, \delta_{i}^{\prime \prime}>0$ are determined in the way that Green's function $G_{\mathrm{M}}(\rho, s ; \lambda)$ is continuous on $\Lambda_{i}$ ). Under $\Lambda_{i}$ we consider a segment of maximum possible length.

It is clear that the maximum value of $Q(\lambda)$ is bounded on each $\Lambda_{i}$. Moreover, by choosing appropriate $\alpha$ the product $\alpha Q(\lambda)$ can be made as small as necessary.

Consider the dispersion equation $\Phi(\lambda)=0$. It is clear that $g(\lambda)$ is continuous and changes its sign when $\lambda$ varies from $\lambda_{i}^{*}-\delta_{i}^{\prime}$ to $\lambda_{i}^{*}+\delta_{i}^{\prime \prime}$. Since $Q(\lambda)$ is bounded on $\Lambda_{i}$, then it is always possible to chose sufficiently small $\alpha$ in order that the 
equation $\Phi(\lambda)=0$ will have at least $m$ roots $\hat{\lambda}_{i} \in\left(\lambda_{i}^{*}-\delta_{i}^{\prime}, \lambda_{i}^{*}+\right.$ $\left.\delta_{i}^{\prime \prime}\right), i=\overline{1, m}$.

The following theorem is the main result of this paper.

Theorem 1. Let $0<\varepsilon_{0}<\min \left(\varepsilon_{1}, \varepsilon_{3}\right)$ and let there be $m \geqslant 1$ solutions $\lambda_{i}^{*}$ of the problem $P_{M}(0)$ such that

$$
\max \left\{\varepsilon_{1}, \varepsilon_{3}\right\}<\lambda_{m}^{*}<\lambda_{m-1}^{*}<\cdots<\lambda_{2}^{*}<\lambda_{1}^{*} .
$$

Then there is $\alpha_{0}>0$ such that for any $\alpha \leqslant \alpha_{0}$ at least $m$ eigenvalues $\widehat{\gamma}_{i}$ of the problem $P_{M}(\alpha)$ exist and $\widehat{\gamma}_{i} \in$ $\left(\sqrt{\lambda_{i}^{*}-\delta_{i}^{\prime}}, \sqrt{\lambda_{i}^{*}+\delta_{i}^{\prime \prime}}\right), i=\overline{1, m}$.

Proof. Let $\Lambda:=\bigcup_{i=1}^{m} \Lambda_{i}$. Green's function $G_{\mathrm{M}}(\rho, s ; \lambda)$ exists for all $\lambda \in \Lambda$. Clearly, the function $A(\lambda)=$ $2 / 3\|\mathbf{h}(\lambda)\| \sqrt{3\|\mathbf{N}(\lambda)\|}$ is continuous with respect to $\lambda \in \Lambda$. Let $A_{1}=\min _{\lambda \in \Lambda} A(\lambda)$ and $\alpha<A_{1}^{2}$. According to Statement 3 there is a unique solution $\mathbf{u}=\mathbf{u}(\lambda)$ to (37) for any $\lambda \in \Lambda$. This solution is continuous and $\|\mathbf{u}\| \leqslant r_{*}=r_{*}(\lambda)$. Let $r_{0}=$ $\max _{\lambda \in \Lambda} r_{*}(\lambda)$. Estimating $Q(\lambda)$ one obtains $|Q(\lambda)| \leqslant C r_{0}^{3}$, where $C$ is a constant.

The function $g(\lambda)$ is continuous and equation $g(\lambda)=0$ has at least one root $\lambda_{i}^{*} \in \Lambda_{i}$. Let $M_{1}=\min _{\lambda \in \Lambda}|g(\lambda)|, M_{2}=$ $\min _{\lambda \in \Lambda}|g(\lambda)|$. Then the value $\widetilde{M}=\min \left\{M_{1}, M_{2}\right\}$ is positive and does not depend on $\alpha$.

If $\alpha \leqslant \widetilde{M} / C r_{0}^{3}$, then for each $\lambda_{i}^{*}$ there exist $\lambda_{i}^{\prime}$ and $\lambda_{i}^{\prime \prime}$ such that $\lambda_{i}^{*}-\delta_{i}^{\prime}<\lambda_{i}^{\prime}<\lambda_{i}^{*}<\lambda_{i}^{\prime \prime}<\lambda_{i}^{*}+\delta_{i}^{\prime \prime}$ and it is true that

$$
\left(C_{1} g\left(\lambda_{i}^{\prime}\right)-\alpha Q\left(\lambda_{i}^{\prime}\right)\right) \cdot\left(C_{1} g\left(\lambda_{i}^{\prime \prime}\right)-\alpha Q\left(\lambda_{i}^{\prime \prime}\right)\right)<0 .
$$

Since the function $\Phi(\lambda)$ is continuous, then the equation $\Phi(\lambda)=0$ has a root $\hat{\lambda}_{i}$ inside $\Lambda_{i}$; to be more precise $\lambda_{i}^{\prime}<\hat{\lambda}_{i}<$ $\lambda_{i}^{\prime \prime}$. We can choose $\alpha_{0}=\min \left\{A_{1}^{2}, \widetilde{M} / C r_{0}^{3}\right\}$.

It follows from this theorem that there exist axisymmetric guided TM-polarized waves without attenuation in circular cylindrical dielectric waveguides filled with nonmagnetic isotropic medium with Kerr nonlinearity. This result generalizes the well-known corresponding assertion for homogeneous dielectric waveguides [17] and for inhomogeneous dielectric waveguides [30] filled with a linear medium (when $\alpha=0)$.

Note 5. An estimation for $\alpha_{0}$ is given in the proof of Theorem 1.

\section{Numerical Results}

We use abbreviations "LP" for "linear problem" and "NLP" for "nonlinear problem."

For calculations a numerical method suggested in $[7,16]$ was used. The following nonlinearities and inhmogeneities were considered.

(1) Kerr law: $\varepsilon=\varepsilon_{2}(\rho)+\alpha u^{2}$ :
(a) $\varepsilon_{2}(\rho)=\varepsilon_{c}+1 / \rho$,
(b) $\varepsilon_{2}(\rho)=\varepsilon_{c}$,
(c) $\varepsilon_{2}(\rho)=\varepsilon_{c}+\rho$

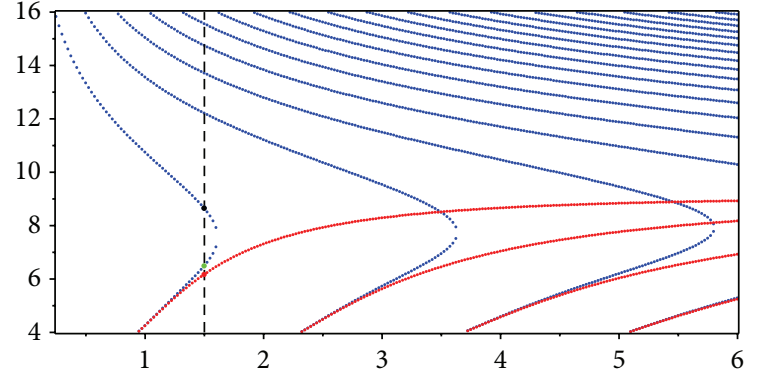

Figure 2: Case 1(a). Marked eigenvalues: $\tilde{\gamma} \approx 2.482$ (red dot, LP), $\widehat{\gamma} \approx 2.546$ (green dot, NLP), and $\widehat{\gamma} \approx 3.940$ (black dot, NLP).

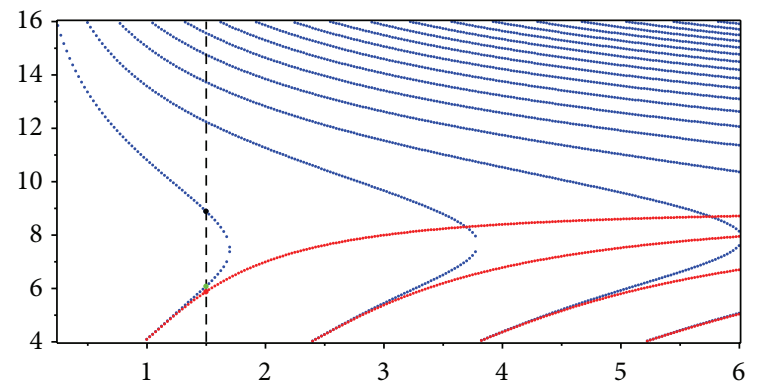

FIGURE 3: Case 1(b). Marked eigenvalues: $\tilde{\gamma} \approx 2.422$ (red dot, LP), $\widehat{\gamma} \approx 2.464$ (green dot, NLP), and $\hat{\gamma} \approx 3.980$ (black dot, NLP).

(2) Law with saturation: $\varepsilon=\varepsilon_{2}(\rho)+\alpha u^{2} /\left(1+\beta u^{2}\right)$ :
(a) $\varepsilon_{2}(\rho)=\varepsilon_{c}+1 / \rho$
(b) $\varepsilon_{2}(\rho)=\varepsilon_{c}$,
(c) $\varepsilon_{2}(\rho)=\varepsilon_{c}+\rho$.

The term $\varepsilon_{c}$ is a positive real constant; we use $\varepsilon_{c}>$ $\max \left\{\varepsilon_{1}, \varepsilon_{3}\right\}$. (In fact, cases 1(b) and 2(b) are homogeneous.)

In the figures the dependence $\gamma \equiv \gamma(\Delta R)$ (Figures 2-4, 1113) and eigenfunctions $u_{1}(\rho), u_{2}(\rho)$ (Figures 5-10, 14-19) are plotted. We call the dependence $\gamma(\Delta R)$ the dispersion curve (DC).

The following parameters were used for the calculations: $\varepsilon_{1}=4, \varepsilon_{c}=9, \varepsilon_{3}=1, R_{1}=2,2<R_{2}<8, k_{0}=1$, and $C_{1}=1$; for the Kerr nonlinearity $\alpha=0.01$ (Figures 2-10).

Dispersion curves for the cases $1(\mathrm{a}, \mathrm{b}, \mathrm{c}), 2(\mathrm{a}, \mathrm{b}, \mathrm{c})$ are shown in Figures 2-4, 11-13, respectively.

In Figures 2-4, 11-13 the vertical axis corresponds to $\gamma^{2}>$ 4 , and the horizontal axis corresponds to the thickness $0<$ $\Delta R=R_{2}-R_{1}<6$ of the cylindrical cladding (see Figure 1 ).

Red lines in Figures 2-4, 11-13 correspond to the linear inhomogeneous case, and blue curves correspond to the nonlinear inhomogeneous case (in the same figure the inhomogeneity is the same for both types of curves).

There is a vertical dashed line in Figures 2-4, 11-13. Eigenvalues (or PCs) of a particular problem are points of intersections of this dashed line with the dispersion curves; first three points in Figures 2-4 are marked (the smallest value, red dot, corresponds to the LP and the other two (green and black dots) correspond to the Kerr case); first four points 


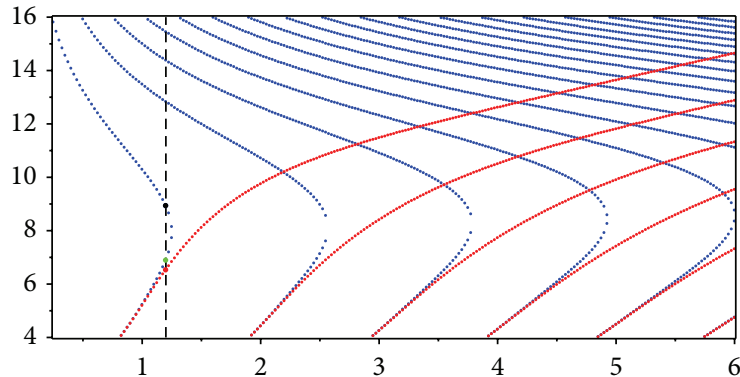

FIGURE 4: Case 1(c). Marked eigenvalues: $\tilde{\gamma} \approx 2.554$ (red dot, LP), $\hat{\gamma} \approx 2.624$ (green dot, NLP), and $\widehat{\gamma} \approx 3.988$ (black dot, NLP).

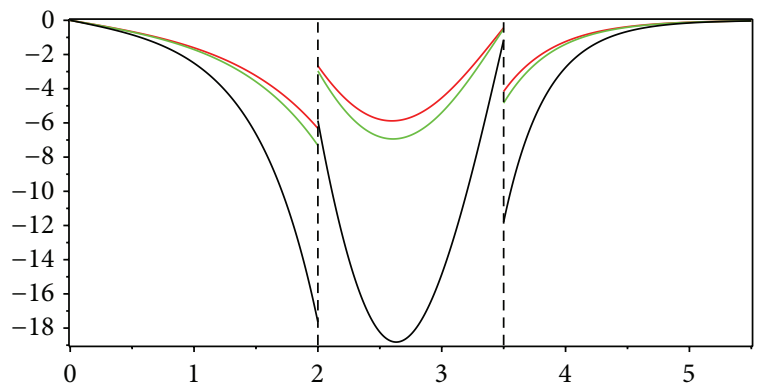

Figure 5: Case 1(a): eigenfunctions $u_{1}(\rho)\left(R_{1}=2, R_{2}=3.5\right)$. LP: $\tilde{\gamma} \approx 2.482$ (red); NLP: $\widehat{\gamma} \approx 2.546$ (green), $\widehat{\gamma} \approx 2.940$ (black).

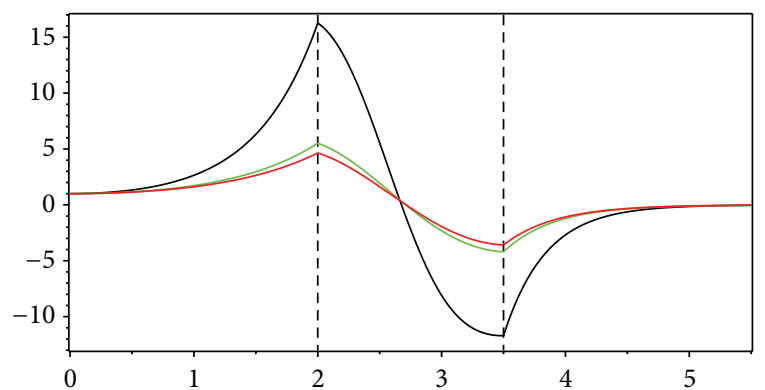

FIgURE 6: Case 1(a): eigenfunctions $u_{2}(\rho)\left(R_{1}=2, R_{2}=3.5\right)$. LP: $\tilde{\gamma} \approx 2.482$ (red); NLP: $\widehat{\gamma} \approx 2.546$ (green), $\widehat{\gamma} \approx 2.940$ (black).

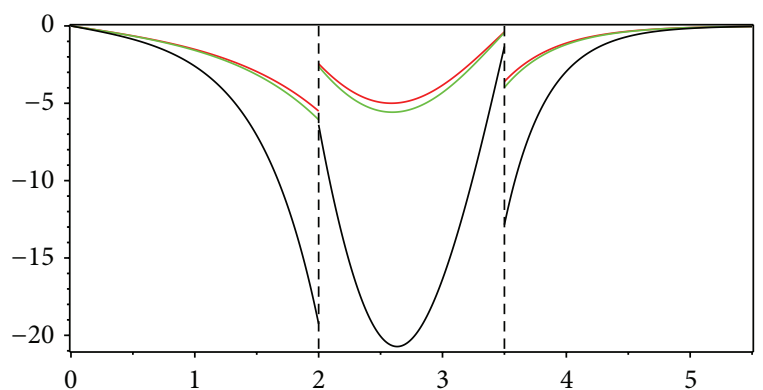

FIGURE 7: Case 1(b): eigenfunctions $u_{1}(\rho)\left(R_{1}=2, R_{2}=3.5\right)$. LP: $\tilde{\gamma} \approx 2.422$ (red); NLP: $\widehat{\gamma} \approx 2.464$ (green), $\widehat{\gamma} \approx 2.980$ (black).

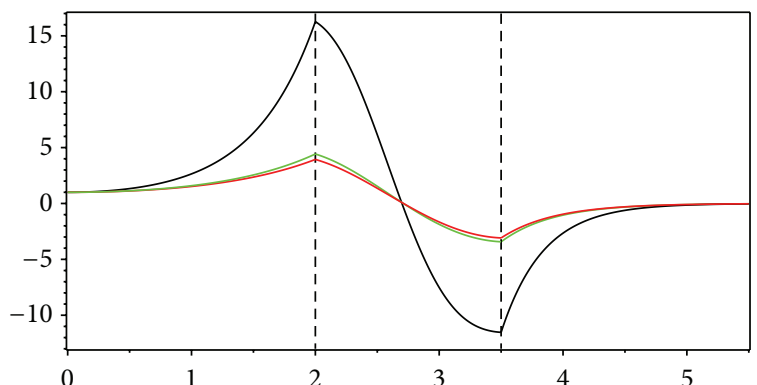

FIGURE 8: Case 1(b): eigenfunctions $u_{2}(\rho)\left(R_{1}=2, R_{2}=3.5\right)$. LP: $\tilde{\gamma} \approx 2.422$ (red); NLP: $\widehat{\gamma} \approx 2.464$ (green), $\widehat{\gamma} \approx 2.980$ (black).

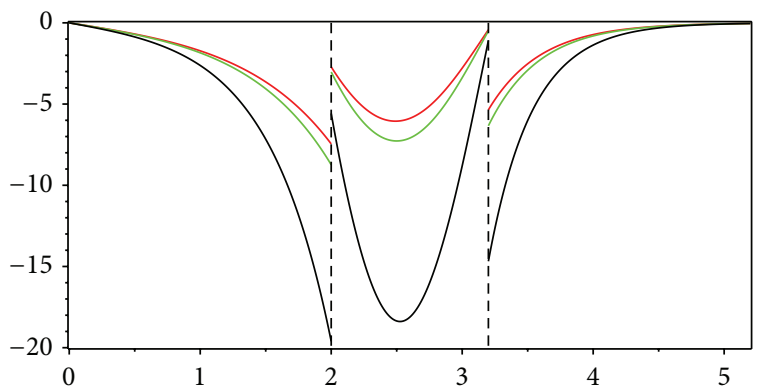

Figure 9: Case 1(c): eigenfunctions $u_{1}(\rho)\left(R_{1}=2, R_{2}=3.2\right)$. LP: $\widetilde{\gamma} \approx 2.554$ (red); NLP: $\widehat{\gamma} \approx 2.624$ (green), $\widehat{\gamma} \approx 2.988$ (black).

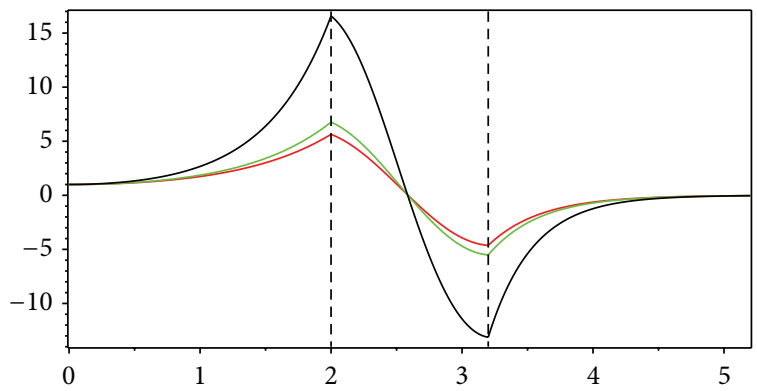

Figure 10: Case 1(c): eigenfunctions $u_{2}(\rho)\left(R_{1}=2, R_{2}=3.2\right)$. LP: $\widetilde{\gamma} \approx 2.554$ (red); NLP: $\widehat{\gamma} \approx 2.624$ (green), $\widehat{\gamma} \approx 2.988$ (black).

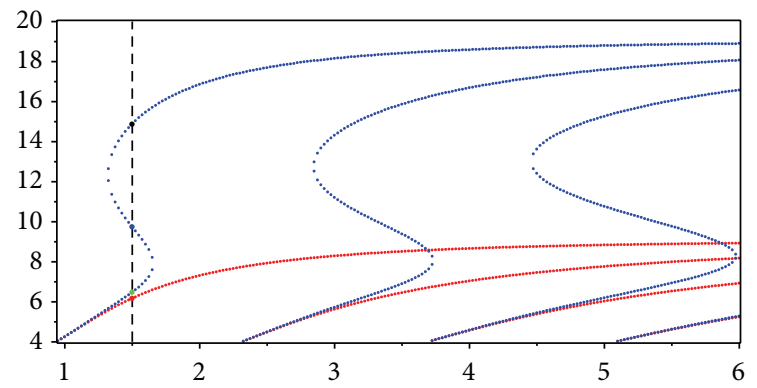

FIgURE 11: Case 2(a). Marked eigenvalues: $\widetilde{\gamma} \approx 2.482$ (red dot, LP), $\widehat{\gamma} \approx 2.5425, \widehat{\gamma} \approx 3.12$, and $\widehat{\gamma} \approx 3.855$ (green, blue, and black dots, resp., NLP). 


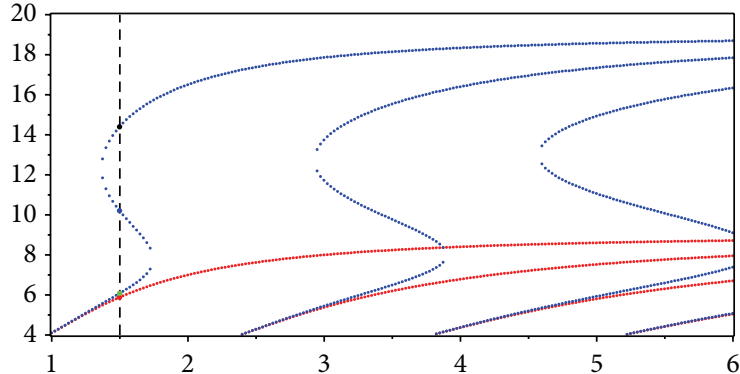

Figure 12: Case 2(b). Marked eigenvalues: $\tilde{\gamma} \approx 2.422$ (red dot, LP), $\widehat{\gamma} \approx 2.4625, \widehat{\gamma} \approx 3.1925$, and $\widehat{\gamma} \approx 3.7925$ (green, blue, and black dots, resp., NLP).

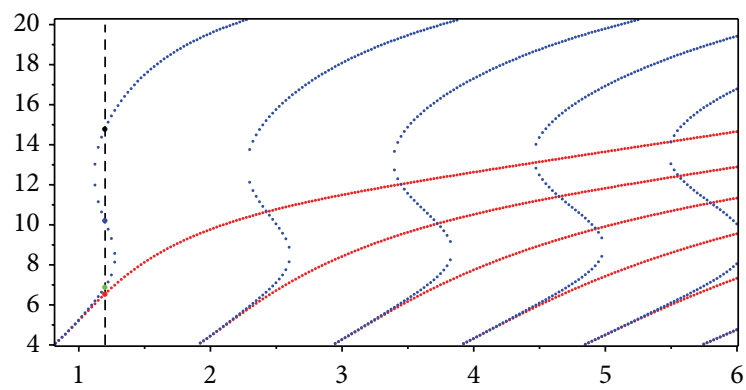

FIGURE 13: Case 2(c). Marked eigenvalues: $\tilde{\gamma} \approx 2.554$ (red dot, LP), $\widehat{\gamma} \approx 2.62, \widehat{\gamma} \approx 3.1925$, and $\widehat{\gamma} \approx 3.8425$ (green, blue, and black dots, resp., NLP).

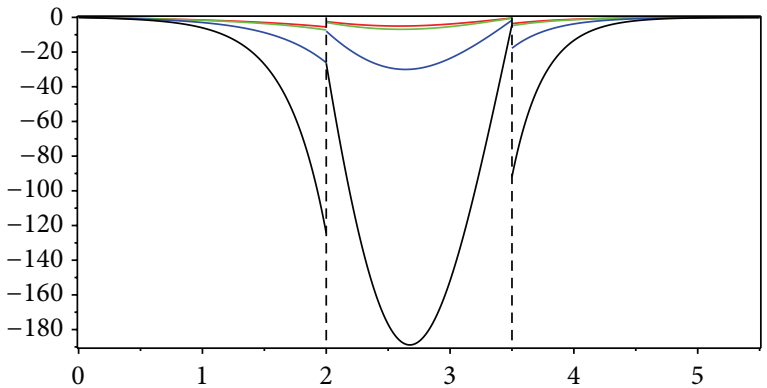

FIGURE 14: Case 2(a): eigenfunctions $u_{1}(\rho)\left(R_{1}=2, R_{2}=3.5\right)$. LP: $\tilde{\gamma} \approx 2.482$ (red); NLP: $\widehat{\gamma} \approx 2.5425$ (green), $\widehat{\gamma} \approx 3.12$ (blue), and $\widehat{\gamma} \approx 3.855$ (black).

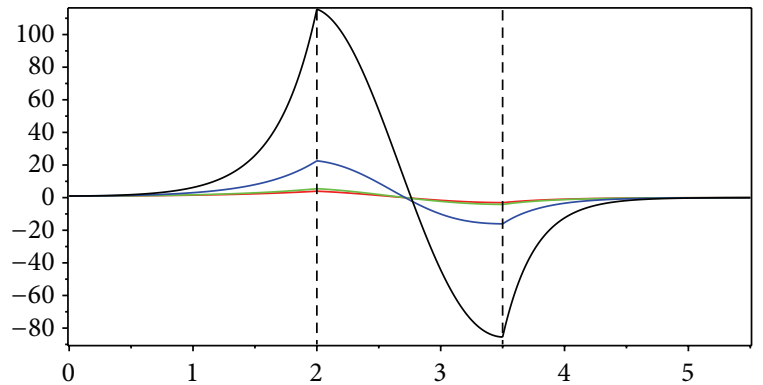

FIgURE 15: Case 2(a): eigenfunctions $u_{2}(\rho)\left(R_{1}=2, R_{2}=3.5\right)$. LP: $\tilde{\gamma} \approx 2.482$ (red); NLP: $\widehat{\gamma} \approx 2.5425$ (green), $\widehat{\gamma} \approx 3.12$ (blue), and $\widehat{\gamma} \approx 3.855$ (black).

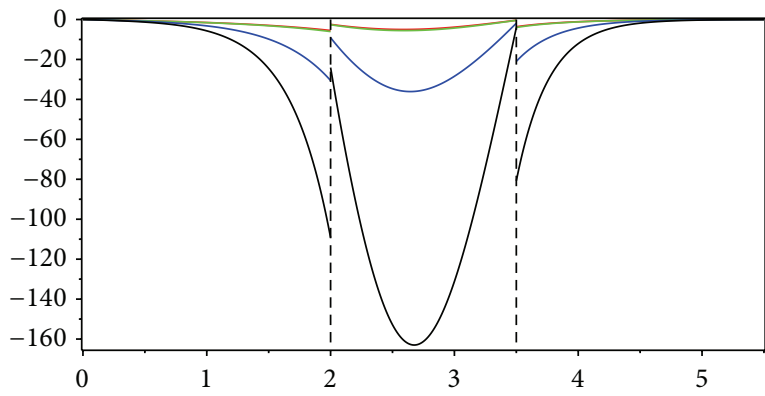

FIgURE 16: Case 2(b): eigenfunctions $u_{1}(\rho)\left(R_{1}=2, R_{2}=3.5\right)$. LP: $\tilde{\gamma} \approx 2.422$ (red); NLP: $\widehat{\gamma} \approx 2.4625$ (green), $\widehat{\gamma} \approx 3.1925$ (blue), and $\widehat{\gamma} \approx 3.7925$ (black)

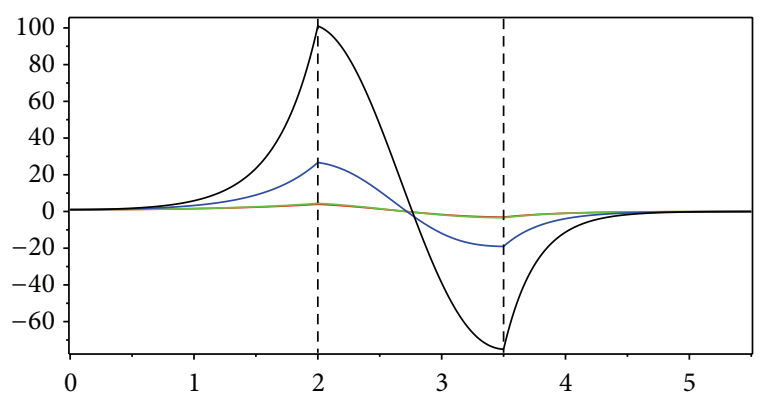

Figure 17: Case 2(b): eigenfunctions $u_{2}(\rho)\left(R_{1}=2, R_{2}=3.5\right)$. LP: $\tilde{\gamma} \approx 2.422$ (red); NLP: $\hat{\gamma} \approx 2.4625$ (green), $\widehat{\gamma} \approx 3.1925$ (blue), and $\widehat{\gamma} \approx 3.7925$ (black).

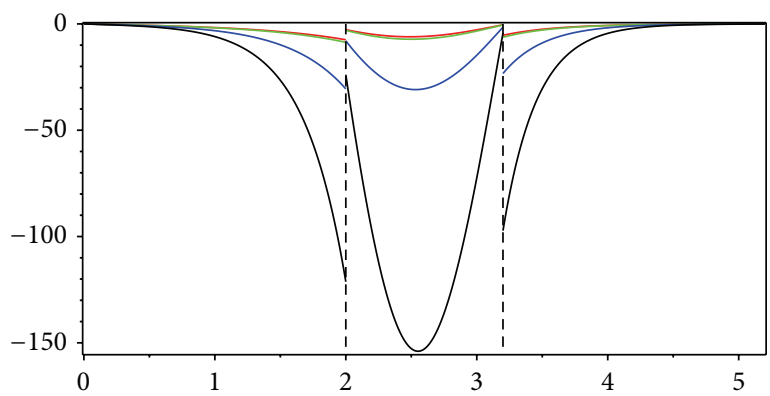

FIgURE 18: Case 2(c): eigenfunctions $u_{1}(\rho)\left(R_{1}=2, R_{2}=3.2\right)$. LP: $\tilde{\gamma} \approx 2.554$ (red); NLP: $\widehat{\gamma} \approx 2.62$ (green), $\widehat{\gamma} \approx 3.1925$ (blue), and $\widehat{\gamma} \approx 3.8425$ (black).

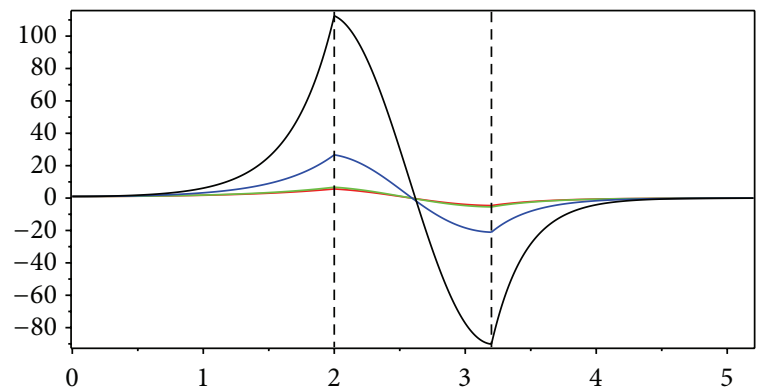

FIGURE 19: Case 2(c): eigenfunctions $u_{2}(\rho)\left(R_{1}=2, R_{2}=3.2\right)$. LP: $\tilde{\gamma} \approx 2.554$ (red); NLP: $\widehat{\gamma} \approx 2.62$ (green), $\hat{\gamma} \approx 3.1925$ (blue), and $\widehat{\gamma} \approx 3.8425$ (black). 
in Figures 11-13 are marked (the smallest value, red dot, corresponds to the LP and the other three (green, blue, and black dots) correspond to the case with a saturated nonlinearity).

Vertical dashed line in Figures 2, 3, and 4 corresponds to $\Delta R=1.5, \Delta R=1.5$, and $\Delta R=1.2$, respectively.

In Figures 5-10, 14-19 the vertical axis corresponds to the value of the plotted functions $u_{1}(\rho), u_{2}(\rho)$ and the horizontal axis corresponds to the value $\rho$.

In Figures 5-10, 14-19 eigenfunctions $u_{1}(\rho), u_{2}(\rho)$ for the cases 1, 2, and 3, respectively, are plotted; the color of a curve corresponds to the color of marked eigenvalue in Figures 2-4, $11-13$.

Vertical dashed line in Figures 11, 12, and 13 corresponds to $\Delta R=1.5, \Delta R=1.5$, and $\Delta R=1.2$, respectively.

\section{Discussion}

Applying the analytical method, which is used in this paper, it is possible to consider an arbitrary continuous nonlinearity with a small multiplier. If one chooses sufficiently small value of the multiplier, then the analytical method allows proving existence of eigenvalues in the nonlinear problem which are close to eigenvalues of the corresponding linear problem. For example, nonlinearities with saturation like $\varepsilon=$ $\varepsilon_{2}(\rho)+\alpha u^{2} /\left(1+\beta u^{2}\right)$ or $\varepsilon=\varepsilon_{2}(\rho)+\alpha\left(1-e^{-\beta u^{2}}\right)[28]$ can also be treated with this method. However, as it is seen from the plots above for different type of nonlinearities, in the nonlinear problems there exist more eigenvalues than Theorem 1 predicts. Up to now the authors are not aware of any rigorous mathematical method which allows solving nonlinear eigenvalue problems of the type considered in this paper (for cylindrical geometry). In other words, it is an open question how to study theoretically this and similar problems $[1,7,12-16]$ completely.

If we compare red (LP) and blue (NLP) dispersion curves in Figures 2-4 we can notice that in LPs there are not more than a finite number of eigenvalues (this fact is well known). In each nonlinear case there are eigenvalues which are close to corresponding linear case (these eigenvalues can be determined with a perturbation theory; existence of these very eigenvalues is proved analytically in this paper). At the same time, it is easy to see, from Figures 2-4, 11-13, that in each nonlinear case there are new eigenvalues which may correspond to a new propagation regime (we call them "purely nonlinear" eigenvalues).

In Figures 2-4 in groups of three eigenvalues are marked. The red dot (smallest value) is an eigenvalue in the linear problem (with $\alpha=0$ ). The green dot is an eigenvalue of the nonlinear problem. When one passes to the limit $\alpha \rightarrow 0$ then the green dot tends to the red dot. Choosing several values of $\alpha, 0<\alpha_{1}<\alpha_{2}<\alpha_{3}$, we did not see from calculations that the black dot tends to an eigenvalue of the linear problem when $\alpha$ decreases; this eigenvalue likely can not be determined with a perturbation theory.

In Figures 11-13 groups of four eigenvalues are marked. The green dot is close to a solution of the linear problem (red dot). Here there are new eigenvalues as well: blue and black dots which also may correspond to a new propagation regime. However, in this case if $\alpha$ decreases, then the new eigenvalues disappear.

Whether these mathematically predicted "purely" nonlinear waves really exist is a hypothesis that can be proved or disproved in an experiment.

It is also important to note that the higher an eigenvalue is, the higher the maximum of the corresponding eigenfunction is (see Figures 5-10, 14-19). Probably, eigenfunctions with sufficiently high maxima correspond to the case in which chosen nonlinear law is no longer valid.

\section{Conflict of Interests}

The authors declare that there is no conflict of interests regarding the publication of this paper.

\section{Acknowledgments}

The authors thank their supervisor Yu. G. Smirnov for his valuable advise. The work is partially supported by RFBR (Project no. 14-01-31234), Russian Federation President Grant (Project no. MK-90.2014.1), and the Ministry of Science and Education of the Russian Federation (Goszadanie, Project no. 2.1102.2014K).

\section{References}

[1] P. N. Eleonskii, L. G. Oganes'yants, and V. P. Silin, "Cylindrical nonlinear waveguides," Soviet Physics-JETP, vol. 35, no. 1, pp. 44-47, 1972.

[2] Y. R. Shen, The Principles of Nonlinear Optics, John Wiley \& Sons, New York, NY, USA, 1984.

[3] L. D. Landau, E. M. Lifshitz, and L. P. Pitaevskii, Course of Theoretical Physics, Volume 8: Electrodynamics of Continuous Media, Butterworth-Heinemann, Oxford, UK, 1993.

[4] W. Man, S. Fardad, Z. Zhang et al., "Optical nonlinearities and enhanced light transmission in soft-matter systems with tunable polarizabilities," Physical Review Letters, vol. 111, no. 21, Article ID 218302, 5 pages, 2013.

[5] L. Ma, J. Hu, M. Costa et al., "Magneto-optical Kerr effect in $\mathrm{L}_{0}$ FePdPt ternary alloys: experiments and first-principles calculations," Journal of Applied Physics, vol. 115, no. 18, Article ID 183903, 6 pages, 2014.

[6] V. Besse, G. Boudebs, and H. Leblond, "Determination of the third- and fifth-order optical nonlinearities: the general case," Applied Physics B: Lasers and Optics, vol. 116, no. 4, pp. 911-917, 2014.

[7] D. V. Valovik and E. Y. Smol'Kin, "Calculation of the propagation constants of inhomogeneous nonlinear double-layer circular cylindrical waveguide by means of the Cauchy problem method," Journal of Communications Technology and Electronics, vol. 58, no. 8, pp. 762-769, 2013.

[8] Y. G. Smirnov and D. V. Valovik, Electromagnetic Wave Propagation in Nonlinear Layered Wave Guide Structures, Penza State University Press, Penza, Russia, 2011.

[9] A. D. Boardman, P. Egan, F. Lederer, U. Langbein, and D. Mihalache, "Third-order nonlinear electromagnetic TE and TM 
guided waves," in Nonlinear Surface Electromagnetic Phenomena, H.-E. Ponath and G. I. Stegeman, Eds., Elsevier Science, 1991.

[10] H. W. Schürmann, V. S. Serov, and Y. V. Shestopalov, “TEpolarized waves guided by a lossless nonlinear three-layer structure," Physical Review E: Statistical Physics, Plasmas, Fluids, and Related Interdisciplinary Topics, vol. 58, no. 1, pp. 1040-1050, 1998.

[11] Y. G. Smirnov and D. V. Valovik, "Nonlinear effects of electromagnetic TM wave propagation in anisotropic layer with Kerr nonlinearity," Advances in Mathematical Physics, vol. 2012, Article ID 609765, 21 pages, 2012.

[12] S. N. Kupriyanova and Y. G. Smirnov, "Propagation of electromagnetic waves in cylindrical dielectric waveguides filled with a nonlinear medium," Computational Mathematics and Mathematical Physics, vol. 44, no. 10, pp. 1850-1860, 2004.

[13] P. N. Eleonskii and V. P. Silin, "Propagation of electromagnetic waves in an inhomogeneous nonlinear medium," Journal of Experimental and Theoretical Physics, vol. 39, no. 1, pp. 67-70, 1974.

[14] H. W. Schürmann, Y. Smirnov, and Y. Shestopalov, "Propagation of TE waves in cylindrical nonlinear dielectric waveguides," Physical Review E-Statistical, Nonlinear, and Soft Matter Physics, vol. 71, no. 1, Article ID 016614, 2005.

[15] Y. G. Smirnov and D. V. Valovik, "On the problem of electromagnetic waves propagating along a nonlinear inhomogeneous cylindrical waveguide," ISRN Mathematical Physics, vol. 2013, Article ID 184325, 7 pages, 2013.

[16] Y. G. Smirnov, E. Y. Smol'kin, and D. V. Valovik, "Nonlinear double-layer Bragg waveguide: analytical and numerical approaches to investigate waveguiding problem," Advances in Numerical Analysis, vol. 2014, Article ID 231498, 11 pages, 2014.

[17] G. I. Veselov and S. B. Raevskii, Layered Metal-Dielectric Waveguides, Radio isvyaz', Moscow, Russia, 1988 (Russian).

[18] J. D. Chatterton and J. L. Shohet, "Guided modes and loss in a plasma-filled Bragg waveguide," Journal of Applied Physics, vol. 102, no. 6, Article ID 063304, 2007.

[19] Y. G. Rapoport, A. D. Boardman, V. V. Grimalsky, V. M. Ivchenko, and N. Kalinich, "Strong nonlinear focusing of light in nonlinearly controlled electromagnetic active metamaterial field concentrators," Journal of Optics, vol. 16, no. 5, Article ID 055202, 2014.

[20] D. V. Valovik, "On the problem of nonlinear coupled electromagnetic transverse-electric-transverse magnetic wave propagation," Journal of Mathematical Physics, vol. 54, no. 4, Article ID 042902, 2013.

[21] Y. G. Smirnov and D. V. Valovik, "Coupled electromagnetic transverse-electric-transverse magnetic wave propagation in a cylindrical waveguide with Kerr nonlinearity," Journal of Mathematical Physics, vol. 54, no. 4, Article ID 043506, 22 pages, 2013.

[22] Y. G. Smirnov and D. V. Valovik, "Problem of nonlinear coupled electromagnetic TE-TE wave propagation," Journal of Mathematical Physics, vol. 54, no. 8, Article ID 083502, 2013.

[23] L. A. Vainstein, Electromagnetic Waves, Radio Isvyaz, Moscow, Russia, 1988, (Russian).

[24] M. J. Adams, An Introduction to Optical Waveguides, John Wiley \& Sons, Chichester, UK, 1981.

[25] E. Kamke, Differentialgleichungen Lösungsmethoden und Lösungen, Gewöhnliche Differentialgleichungen, Akademische Verlagsgesellschaft Geest \& Portig K.-G., Leipzig, Germany, 1959.
[26] D. V. Valovik, "Integral dispersion equation method to solve a nonlinear boundary eigenvalue problem," Nonlinear Analysis: Real World Applications, vol. 20, no. 12, pp. 52-58, 2014.

[27] M. Abramowitz and I. A. Stegun, Handbook of Mathematical Functions, National Bureau of Standards, Washington, DC, USA, 1972.

[28] N. N. Akhmediev and A. Ankevich, Solitons, Nonlinear Pulses and Beams, Chapman \& Hall, London, UK, 1997.

[29] Y. G. Smirnov, “Transmission eigen value problems to describe electromagnetic te and tm wave propagation in two-layered inhomogeneous anisotropic cylindrical and plane wave guides," Computational Mathematics and Mathematical Physics. In press.

[30] A. S. Zil'bergleit and Y. I. Kopilevich, Spectral Theory of Regular Waveguides, FTI, Leningrad, Russia, 1983, (Russian). 


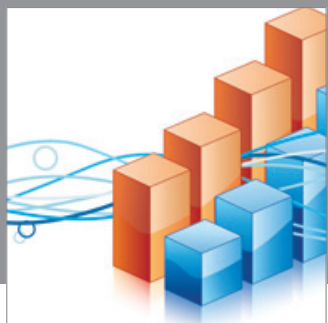

Advances in

Operations Research

mansans

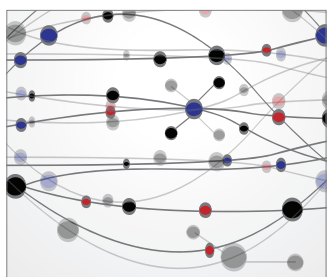

The Scientific World Journal
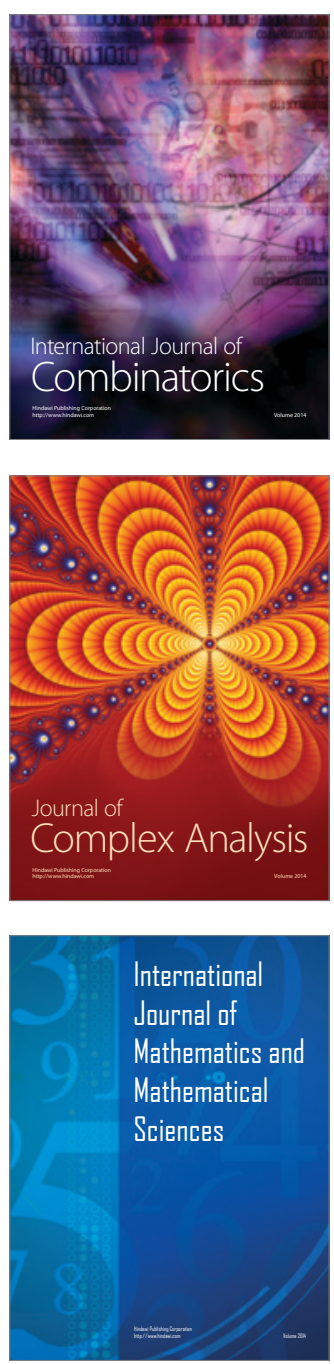
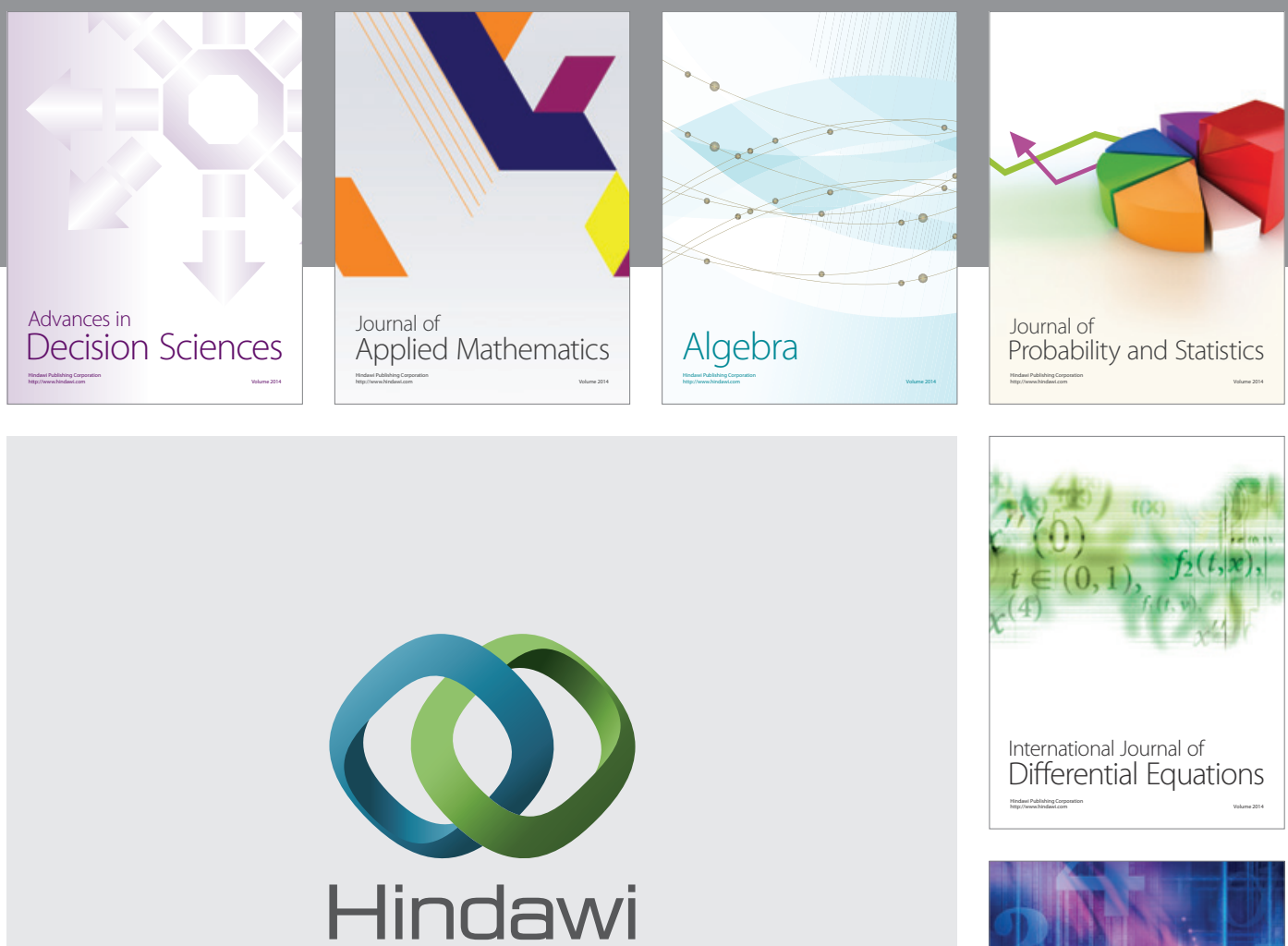

Submit your manuscripts at http://www.hindawi.com
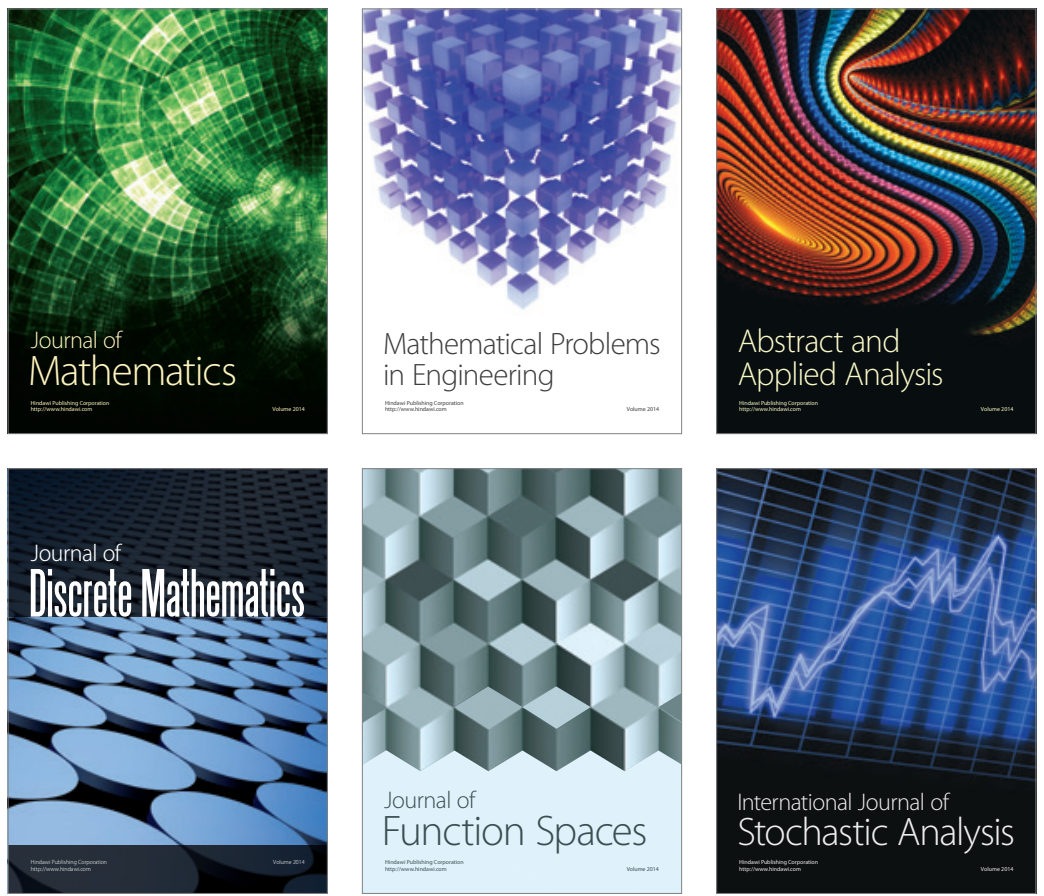

Journal of

Function Spaces

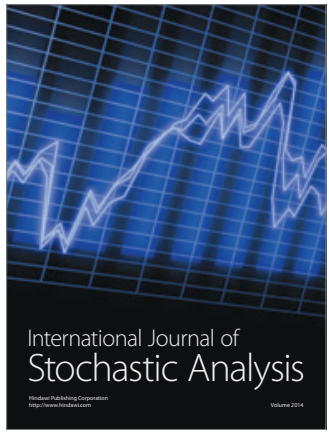

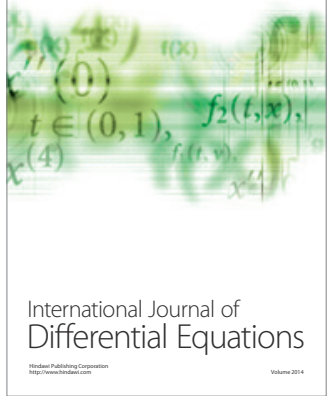
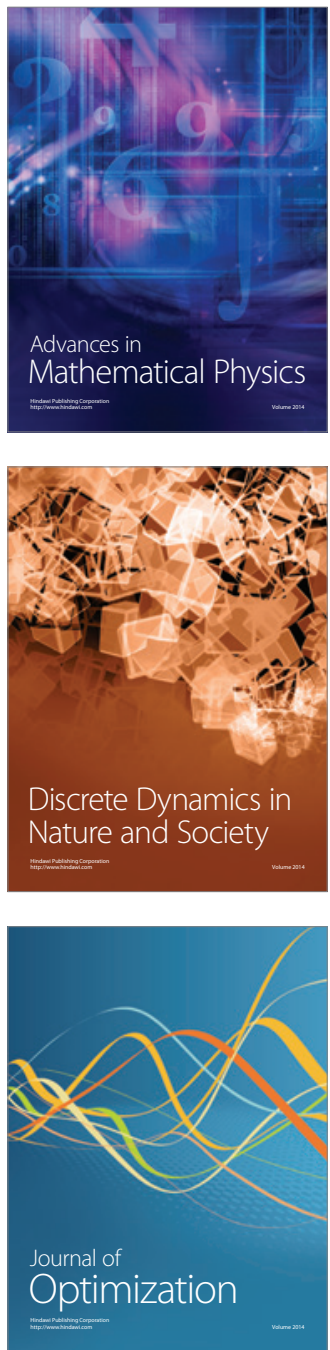